\title{
Analysis of the Effectiveness of an Intervention on Good Practices in Food Handling in Kitchens of the Municipality of Araucária
}

\author{
Gianna Surek $^{1 *}$ and Katia Zoghbi Ospedal ${ }^{2}$ \\ ${ }^{1}$ Academic of the Specialization Course in Industrial Microbiology and Food (Curitiba, PR), Brazil \\ ${ }^{2}$ Pharmaceutical Biochemistry Professor Deputy of the Specialization Course in Industrial Microbiology and Food, Brazil \\ *Corresponding Author: Gianna Surek, Academic of the Specialization Course in Industrial Microbiology and Food (Curitiba, PR), Brazil.
}

Received: October 29, 2019; Published: November 08, 2019

DOI: $10.31080 /$ ASMI.2019.02.0430

\begin{abstract}
Handlers are the main route of food contamination, which can lead to the outbreak of foodborne diseases (TAD's). The present work aimed to describe the importance of a good practice awareness project in food manipulation and its relationship with the reduction of food contamination risks in kitchens of units of the Secretariat of Social Assistance of the Municipality of Araucaria-PR. A check-list was held according to RDC 216/04 in the places, application of a questionnaire to cooks to evaluate their knowledge on the subject and an educational lecture based on the responses and "non-conformities" noted during the study. The main failures noted were in the following questions: buildings and facilities; equipment, furniture and utensils; manipulators; raw materials and packaging; food preparation; exposure to prepared food consumption and documentation and registration.
\end{abstract}

Keywords: Food Handlers; Good Manufacturing Practices; Food Contamination

\section{Introduction}

The search for a better quality of life has become a concern, increasingly latent and effective in people, and health is directly related to several factors that can contribute to a better life, including food, which has a role fundamental because it contributes to the strengthening of health, but can also cause and transmit diseases [1].

Food security is a necessity for organizations operating in the food segment, since it leads to not generating risks to consumer health. Strict supervision should be carried out at all stages of production, not only in the final production, but at all stages, through transport, storage and processing, until the final distribution to the consumer [2].

Food can contain microorganisms known as pathogens, which can cause diseases to the consumer. The most common symptoms include nausea, stomach pain, vomiting, diarrhea and fever, how- ever, depending on the etiological agent and involved, the clinical picture can be extremely serious, with severe dehydration, bloody diarrhea, insufficiency renal failure [3].

For the prevention of these diseases, the education and training of handlers working in food services for the incorporation of practices aimed at quality control and food safety $[4,5]$.

Intervention in education for proper food manipulation can help maximize the safety of the handler in food handling, expand the educational perspectives of this and provide the population with a safe food from the point of view microbiological, physical and chemical [6].

One way to educate the manipulator is to make him know how potentially transmitting microorganisms of food borne diseases act on the human host and what should be done to offer safe foods from a microbiological point of view. Several pathogens have common interaction mechanisms with the host, although each species 
has developed a unique strategy that enables it to exploit the eukaryotic cell [7].

There are approximately 250 types of eating diseases and, among them, many are caused by pathogenic microorganisms, which are responsible for serious public health problems and significant economic losses. The syndromes resulting from the ingestion of food contaminated by these microorganisms are known as Food Borne Diseases (DTA), Food Borne diseases (VAD) or simply to infections [8].

Another important concept in ensuring healthy food is that of "hazards", causing DTAs, which can be of chemical, physical or biological origin [9].

All foods have chemicals in their constitution. Chemical hazards can have a natural origin, such as mycotoxins, histamine, plant toxins, algae or mollusks, toxic mushroom species, etc. or be accidentally introduced during the process, such as pesticides, heavy metals, veterinary medicines, nitrites, nitrates and nitrosamines, lubrication chemicals, cleaning agents, paints and coatings, among others. When ingested at high doses they are responsible for sudden diseases of great intensity, such as poison poisoning. But they are also responsible for chronic diseases when ingestion is done in small lifelong doses, such as carcinogenic or accumulative substances [10].

In the category of physical hazards includes a vast set of dangers with diverse origins, from objects that may be present in raw materials to objects that can be introduced into food through their manipulation. These objects introduced during the process also have several origins, can come from the packaging materials and packaging of raw materials, equipment and utensils and also from the operators themselves. The most frequent physical hazards encountered are: glasses, woods, stones, metals, insulation or coating materials, plastics and objects of personal use. They pose a high risk to the life of the consumer [11].

Biological hazards are those that pose the greatest risk to food safety. In this category are includes bacteria, fungi, viruses and pathogenic parasites and microbial toxins. These organisms are often associated with food manipulation by operators and contaminated raw products that are used as raw materials. Many of these microorganisms occur naturally in the environments where these foods are produced. Several are destroyed by thermal pro- cesses and many can be controlled through appropriate handling and storage practices, good hygiene practices and control of time and temperature processes [11].

When the agent is a toxin previously elaborated by a given microorganism in food, the disease is called toxynose. Thus, viable cells do not need to be present for the disease to occur. Therefore, when there is the intake of viable cells of the pathogenic microorganism, colonization and/or invasion, the disease is called "food infection". When colonization and action of toxins occur, the disease is called "food toxification", diseases caused by Bacillus cereus and Clostridium perfringens (EMBRAPA)are examples of toxifications.

Salmonella is one of the most involved enteropathogenesis in cases and outbreaks of food origin in several countries, including Brazil. Salmonella infections have been associated with a variety of foods, with eggs, poultry meats, pigs, cattle and vegetables the most frequent. Infection caused by this bacterium causes nausea and vomiting, abdominal pain and fever [12].

Another very common bacteria causing infection is Escherichiacoli. It causes abdominal pain, aqueous or bloody diarrhea, fever, nausea and vomiting. Symptoms vary depending on the strain causing infection and incubation period. Virtually all food or plant origin or of animal origin that have not been processing objects, can vehicular E. coli, provided that at some point they have been subjected to fecal pollution $[13,14]$.

Staphylococci are also commonly linked to food poisoning. Its nutritional versatility and the ability to grow in different environmental conditions cause Staphylococcus aureus, the main species of this genus, to develop easily in food. Regarding symptomatology, the incubation period and the onset of symptoms vary according to individual sensitivity and the amount of toxin in the ingested food. The main symptoms are nausea, vomiting, abdominal cramps and diarrhea [15].

According to data from the Hospital Information System (SIH) of the Ministry of Health, there were more than 3,400,000 hospitalizations per TAD in Brazil from 1999 to 2004, with an average of about 570,000 cases per year. And according to the Mortality Information System (SIM), from 1999 to 2002, there were 25,281 deaths from TAD in Brazil, with an average of 6,320 deaths per year [3]. 
The concern with food quality has motivated the development and creation of regulations and tools as a way to guarantee the consumer what they seek and meet the demands of marketing. Among the tools used by organizations are good handling practices (BPM) [16].

BPM are hygiene practices that must be obeyed by handlers from the choice and purchase of products to be used in the preparation of food to the sale to the consumer. The objective is to avoid the occurrence of diseases caused by the consumption of contaminated food [17].

On October 21, 2002, The Resolution of the Collegiate Board No. 275 was created, which provides on the Technical Regulation of Standardized Operational Procedures (POP's) applied to Food Producing/Industrializing Establishments and the List of Verification of Good Manufacturing Practices in Food Producing/Industrializing Establishments [18].

This resolution was created in view of the need for constant improvement of health control actions in the food area aimed at protecting the health of the population; considering the need to harmonize the health inspection action in food-producing/industrializing establishments throughout the national territory; considering the need to complement the Technical Regulation on Hygienic-Sanitary Conditions and Good Manufacturing Practices for Food Producing/Industrializing Establishments; considering the need to development of a generic instrument for verifying Good Manufacturing Practices applicable to food producing/industrializing establishments [18].

To ensure that the establishments are complying with the necessary procedures for the manufacture/production of a safe food, the Ministry of Health, through ANVISA also prepared Resolution - RDC No. 216, of September 15, 2004, which provides on Technical Regulation of Good Practices for Food Services. This resolution applies to food services that carry out some of the following activities: handling, preparation, fractionation, storage, distribution, transportation, exposure to sale and delivery of food prepared for consumption, such as canteens, buffets, commissaries, confectioneries, industrial kitchens, institutional kitchens, delicatessens, snack bars, bakeries, pastries, restaurants, rotisserie and congeners [19].
DRC 216 aims to ensure sanitary hygienic conditions for food, involving actions in the areas of building, facilities, equipment, furniture and utensils, hygiene and maintenance; pest and vector control; water supply; waste management; health of handlers; raw materials, ingredients and packaging; pre-preparation until exposure to prepared food consumption [19-21].

The aim of this article is to describe the importance of a project to raise awareness of Good Food Manipulation Practices and its relationship with reducing the risks of food contamination.

\section{Materials and Methods}

The preparation of the proposal for an intervention on good food manipulation practices was carried out in kitchens of the Secretariat of Social Assistance of the Municipality of AraucariaPR, which included two houses of the harvest, a house destined to homeless people and a living center for the elderly.

The proposed methodology was based on actions to promote education in Good Food Manipulation Practices to the four cooks of the respective units studied.

This methodology was divided into four phases:

First phase: A check-list was performed without prior scheduling between $9 \mathrm{am}$ and $1 \mathrm{pm}$ on a single visit, from 05/08 to $20 / 05 / 2019$, with the objective of evaluating compliances/nonconformities, so that general aspects of personal hygiene and training program; general aspects of projects and facilities; general aspects of manipulation; general aspects of cleanliness and sanitization; general aspects of integrated pest control and general aspects of quality control, according to RESOLUTION RDC No. 216 of September 15, 2004. This check list consisted of a table divided into eight parts referring to the aspects mentioned above in the following order:

1. Buildings and facilities.

2. Equipment, furniture and utensils.

3. Manipulators.

4. Raw materials, ingredients and packaging.

5. Food preparation.

6. Storage and transportation of prepared food.

7. Exposure to the consumption of prepared food.

8. Documentation and registration. 
For each part there are three columns, in which the researcher observed on site the questions and subsequently pointed out the appropriate response to the evaluation of that issue using the indicators: $\mathrm{S}$ for Yes, when there was compliance with the resolution, $\mathrm{N}$ to No, when there was no compliance with the resolution, and NA when it does not apply to that matter.

Second phase: Application of a questionnaire which was created and applied by the researcher herself in person on the same days of the first phase described above, in order to verify the quantitative and qualitative level of the information that cooks had regarding "Good Practices". This investigation protocol contained 10 questions, with answer options "yes" and "no", in addition to the open justification for the answer given.

The questions of this investigation protocol will be presented below:

1- Do you know/have had training on Good Manufacturing Practices?

() Yes

() No

2- Do you know how food contamination occurs?

() Yes

() No

If so, what are the main causes?

3- Do you know any diseases caused by food contamination?

() Yes

() No

If so, which one?

4- Sanitizes all the equipment you use in food handling/preparation?

() Yes

() No

If so, when is this hygiene one?

Which product is used to perform this cleaning?
5- Does peis (Personal Protective Equipment) use during food handling?

() Yes

() No

If so, which ones?

6- Is there a pest and vector control in the unit?

() Yes

() No

If so, who does?

When do you do it?

7- Wash hands carefully every time you're going to manipulate a food?

() Yes

() No

8- Do you know the difference between recycled waste and organic waste?

() Yes

() No

If so, which one?

9- Do installations (floor, wall, ceiling, windows, doors) interfere in food quality?

() Yes

() No

If so, why?

10- Does it confer all the validities and general aspects of the food received?

() Yes

() No 
Third phase: It consisted of an educational lecture elaborated and presented by the researcher considering the "Good Practices of Food Manipulation", according to Resolution RDC No. 216, of September 15, 2004, as well as, also based on the answers obtained in the interviews, in addition to the "nonconformities" observed by the researcher during the checklist. This lecture aimed at the cooks of the Municipal Secretariat of Social Assistance of the Municipality of Araucaria in an expository way with the objective of raising awareness and teaching the "Good Practices of Food Manipulation" for these professionals. The lecture was held on 02/07/2019, starting at 13:15 pm and lasting approximately 50 minutes.

Fourth phase: Fixing two posters of easy visualization and understanding in the kitchens, elaborated by the researcher herself, containing the main information and instructions regarding the "Good Handling Practices", which are about the correct use of PPE's (Figure1) (pg-20) and on the correct separation of garbage (Figure 2) (pg-21). They were based on the responses obtained in the interviews and also on the "non-conformities" observed during the other phases of the project.
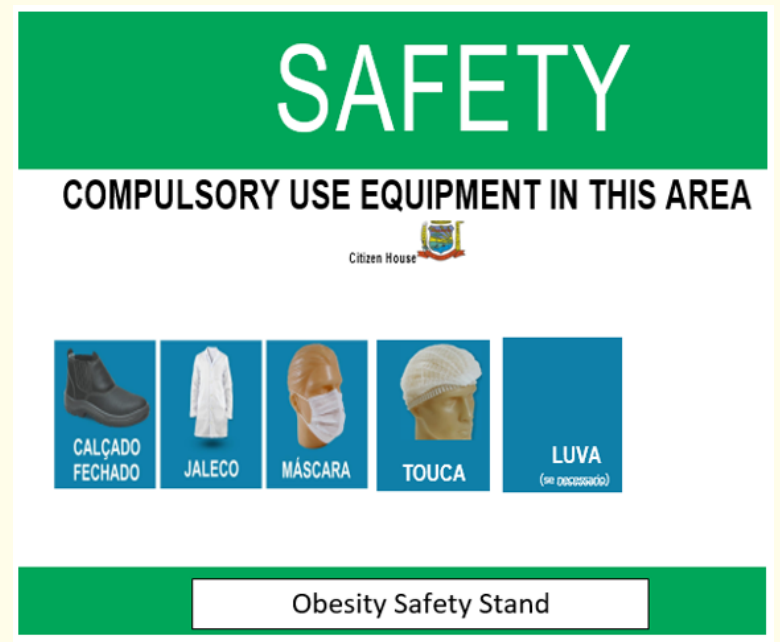

Figure 1: Individual Protective Equipment (PPE's) indispensable in the area of food manipulation.

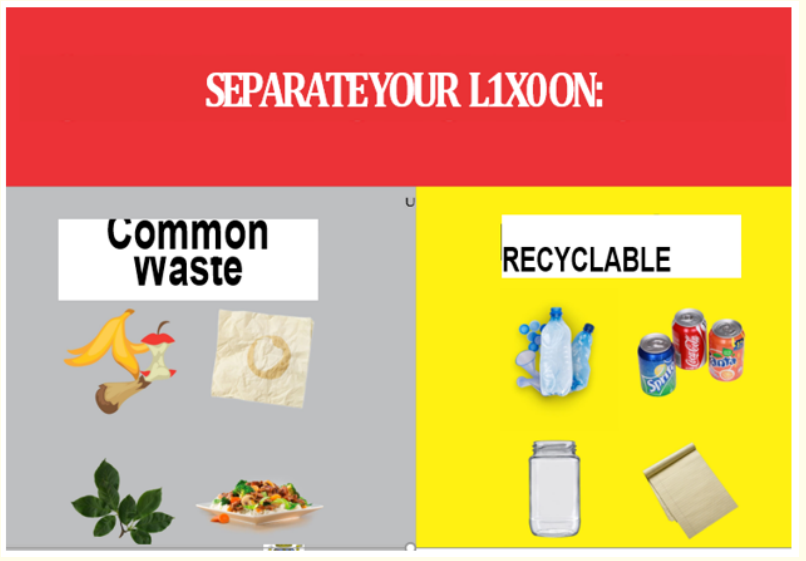

Figure 2: Correct separation of garbage.

\section{Results and Discussion}

In the four kitchens analyzed, some nonconformities were found.

In the Women's Reception House, non-conformities were noted in the following questions:

Building and facilities: PVC lining com infiltrations/peelings, non-closed doors and without millimetric screens, do not have specific washbasins for hand hygiene and also do not have records of cleaning operations performed not routinely.

Mobile equipment and utensils: equipment is not routinely maintained and they were not in a good state of conservation, besides being of unsuitable materials, such as wood for example.

Handlers: There is no record of the handler's health control and nor a periodic training program for it. In addition, it does not use all the PPE needed during food handling.

Raw materials, ingredients and packaging: no temperature check of raw materials and ingredients that require special condition is checked, and storage is done in a very small place, without proper ventilation/lighting, wooden shelves glued to the walls and with inadequate state of conservation. 
Food preparation: Garbage littered with pedal less lids for drive, ingredients opened and stored without the necessary information (product designation, date of fractionation and shelf life), do not have a hood and no monitoring of the temperature of refrigerators and freezers.

Exposure to prepared food consumption: they have only a small stainless steel buffet, with no protection barrier preventing contamination and without routinely checking the temperature.

Documentation and registration: There are no Standardized Operational Procedures (POPs) or a Manual of Good Manufacturing Practices.

In the Men's Reception House, non-conformities were noted in the following questions:

Building and facilities: Ceiling with inadequate coating, unclosed doors of automatic closure and without millimeter screens, no exclusive washbasin for hand hygiene, luminaires not protected against explosions and accidents, waste collectors in toilets not equipped with pedals and only one waste collector for the whole kitchen, also not equipped with pedal, without records of cleaning operations performed not routinely, no cleaning of the fat box is carried out periodically, the structure of the kitchen is very small for the demand it requires and is also located in an inadequate place, having a high flow of people passing through it at all times.

Equipment, furniture and utensils: equipment is not maintained periodically, material of inadequate furniture, most of which are wood without a good state of conservation, presenting cracks and peelings.

Manipulators: there are no posters of guidance for the handler in the kitchen, there is no health control record of the same or a periodic training program. In addition, the handler also does not use all the PPE needed during food preparation.

Raw materials, ingredients and packaging: temperatures are not checked in the reception of products that require special storage conditions and storage place although large is not in good condition.

Food preparation: Only a garbage equipped with a pedal less cover for drive, ingredients opened and stored without the nec- essary information (product designation, date of fractionation and shelf life), do not have a hood and is not made to monitoring the temperature of refrigerators and freezers. It was observed the inadequate thawing of meats and exposure of fruits and vegetables, besides also being located in a place prone to food contamination.

Exposure to prepared food consumption: There is no buffet for children to serve, food is served on a counter without protection barriers and no temperature controlled.

Documentation and registration: There are no Standardized 0perational Procedures (POPs) or a Manual of Good Manufacturing Practices.

The non-conformities observed at the Citizenship House were as follows:

Building and facilities: many objects were noticed in disuse around the kitchen, dark floors with cracks and cracks, ceiling with some cracks and dirt, inadequate state of conservation of sanitary facilities, walls and partitions, unequipped doors manual closure and no millimetric screens, windows also without protective screens, waste collectors without pedal, no specific washbasin for hand hygiene, very dark kitchen, small and muffled, with only one lamp without protection against explosions and accidents and only one window, located on top of the stove. The kitchen presented itself very dirty and disorganized.

Equipment, furniture and utensils: there is no periodic maintenance of equipment, material of inadequate furniture, most of which are wood without a good state of conservation, presenting cracks and peelings.

Manipulators: Clothing not compatible with the activity, does not use all the PPE necessary during food preparation, there are no posters of guidance for the cook, lack of periodic health control and a training program and the visitors do not meet the same requirements as the handlers.

Raw materials, ingredients and packaging: the reception of raw materials and ingredients is carried out in the kitchen itself, without properly inspecting and checking temperatures, poorly stored foods and without proper identification.

Food preparation: containers for collections of unpetalled waste to be triggered without manual contact and in small quantity, after 
opening or removing the original packaging is not made identification with the necessary information of the food, not it has hood, defrosting products performed improperly and the temperature of refrigerators and freezers is not done correctly or monitored.

Exposure to prepared food consumption: there is no specific buffet for this purpose or protective barriers to prevent food contamination.

Documentation and registration: There are no Standardized Operational Procedures (POPs) or a Manual of Good Manufacturing Practices.

\section{And in the ELDERLY HIM}

Center, the non-conformities observed were as follows:

Building and facilities: floor with some cracks, wall and ceiling with improper coating, un-end-of-closed doors and no millimetric screens, windows also without protective screens, there is no record of non-routine cleaning operations and had only one waste collector to suit the whole kitchen.

Manipulators: kitchen presented only one orientation poster, there is no record of health control of them and nor a periodic training program. It does not use all the PPE needed during food preparation.

Raw materials, ingredients and packaging: at food reception, the temperature of those in need of special conservation conditions is not checked.

Food preparation: after opening a food package, the correct identification is not made with the information necessary for storage.

Exposição ao consumo do alimento preparado: não há buffet específico para essa finalidade e nem barreiras de proteção para prevenir a contaminação dos alimentos.

Documentation and registration: There are no Standardized Operational Procedures (POPs) or a Manual of Good Manufacturing Practices.

Regarding the questions asked by the cooks, it was found that all of them have already had a training on Good Manufacturing
Practices, know how food contamination occurs, sanitize all the equipment used in the handling/preparation of food and carefully wash their hands before manipulating them, know the difference between recycled waste and organic waste, believe that the facilities (floor, wall, ceiling, windows, doors) interfere in the quality of a food and confer all validity and general aspects of the food received.

Regarding the question regarding diseases caused by food contamination, three of them answered only the symptoms of diseases, such as vomiting, diarrhea, and only one cited Salmonella as AD.

In question number six, with regard to pest control and vectors in the units studied, it was found that this control is done by a third party company every six months.

From the results obtained, an educational lecture was elaborated for the four cooks, recommending the basic subjects and procedures to be adopted by them, in order to improve the hygienic-sanitary conditions of the places and handlers, based on the recommended by the relevant legislation.

In the lecture, the researcher commented on the work carried out in the units and gave feedback regarding the check-list and the questionnaire answered by the cooks. He also addressed important issues such as the goal and importance of Good Practices in food manipulation, he explained about biological, physical and chemical hazards, spoke about the use of disposable gloves, a process of defrosting meats and other foods, washing fruits and vegetables, hygiene of facilities, personal hygiene, cross-contamination and hand washing.

\section{Conclusion}

According to the results obtained, it can be concluded that changes are necessary in the units studied, both in relation to the physical structure and in relation to the handlers. However, as these are public places, there is resistance to making high-cost investments that favors compliance with all required items. It is also remarkable the need for constant training of cooks. It is suggested, then, a more effective collection of supervisory bodies to minimize the risks of contamination and consequently the incidence of foodrelated diseases. 


\section{Bibliography}

1. Rosa PT. "Implementation of the Manual of Good Handling Practices in pedagogical kitchen of an educational institution in the city of Campo Mourão PR". Course Completion Work (Food Technology) - Academic Department of Food - Federal Technological University of Paraná, Campo Mourão (2016): 176.

2. Valente D and Passos AD. "Hygienic-sanitary and physicalstructural evaluation of supermarkets in a city in South-eastern Brazil". Revista Brasileira de Epidemiology 7.1 (2004): 8087.

3. Welker CAD., et al. "Microbiological analysis of foods involved in outbreaks of foodborne diseases (TAD) that occurred in the state of Rio Grande do Sul, Brazil". Brazilian Journal of Biosciences 8.1 (2010): 44-48.

4. Góes JAW., et al. "Training of food handlers and the quality of food served". Food Hygiene 82 (2001): 20-22.

5. Tavolaro P., et al. "Evaluation of knowledge in hygiene practices: a qualitative approach". Interface Communication, Health, Education 10.19 (2006): 243-254.

6. Levinger B. "School feeding, school reform, and food security: connecting the dots". Food Nutrition Bulletin 26.2 (2005): 170178.

7. Finlay BB and Falkow S. "Commons themes in microbial pathogenicity revisited". Microbiology Molecular Biology Reviews 61.2 (1997): 136-169.

8. Oliveira ABA., et al. "Foodborne diseases, main etiological agents and general aspects: a review". Rev HCPA 30.3 (2010): 279-285.

9. Food Security. Food Ingredients Brasil (2008): 32-43.

10. Afonso A. "Hazard analysis: Identification of hazards and risk assessment for food security". Activate Hygiene and Food Safety 5 (2008): 26-28.

11. Baptista P and Venâncio A. "Hazard classification from a food security perspective. In: The dangers to food safety in food processing". 1the edition. Forvisão, (2003): 10-22.

12. Barros VRM., et al. “. "Salmonella spp: its transmission through food". Food Hygiene Magazine 16.94 (2002): 15-19.

13. Pinto AFMA. "Diseases of microbial origin transmitted by food". Millenium, 4 (1996): 91-100.
14. Nataro JP and Kaper JB. "Diarrheagenic escherichia coli". Clinical Microbiological Reviews 11.1 (1998) 142-201.

15. Santana EHW., et al. "Mendonça, M. B. O. C. Staphylococci in food". Arquivos do Instituto Biológico 77.3 (2010): 545-554.

16. Gonçalves PM and Silva HF. "Good Manufacturing Practices GMP: application in a food packaging industry". RAF - FATEA's Management Journal (2008).

17. Anvisa. "Booklet on Good Practices for Food Services". 3the edition. Brasilia: (2007).

18. Anvisa. "National Health Surveillance Agency". Collegiate Board Resolution - DRC 275 (2002).

19. Anvisa. "National Health Surveillance Agency". Collegiate Board Resolution - DRC 216 (2004).

20. Colombo M., et al. "Knowledge of the merendeiras of Santa Fe, $\mathrm{PR}$, about hygiene and good manufacturing practices in food production". Food Hygiene 23-170/171 (2009): 39-46.

21. Embrapa. Biological hazards (2019).

Volume 2 Issue 12 December 2019 (C) All rights are reserved by Gianna Surek and Katia Zoghbi Ospedal. 